\title{
Hero-Geschichten: Vorbilder für die ganze Gesellschaft
}

\section{Janne Müller-Wieland}

Janne Müller-Wieland ist Hockeyspielerin und Spielführerin der deutschen Hockey-Nationalmannschaft. Neben Titeln als Welt- und Europameisterin (2018 \& 2013) und mehrfache deutsche Meisterin war ibr bislang größter internationaler Erfolg der Gewinn der Bronze-Medaille bei den Olympischen Spielen 2016 in Rio de Janeiro. Dafür wurde sie mit ihrem Team mit dem Silbernen Lorbeerblatt ausgezeichnet.

Um den olympischen Traum zu leben, benötigt es mehr als sportliche Höchstleistung. Als Spitzensportlerin in Deutschland bin ich mit vielen verschiedenen Herausforderungen und Spannungsfeldern konfrontiert. Ich muss nicht nur sportliche Hürden meistern, sondern mich auch immer wieder mit meiner Zukunft nach dem Sport auseinandersetzen und gleichzeitig aushalten, dass viele Menschen meinen Lebensinhalt die meiste Zeit sehr kritisch betrachten. Dennoch würde ich mich immer wieder für meinen Weg und den Leistungssport entscheiden - ich wäre sonst nie die geworden, die ich bin.

Dieser Weg hat mich dreimal zu Olympischen Spielen geführt: 2008 in Peking, 2012 in London und 2016 in Rio de Janeiro konnte ich sehr unterschiedliche Spielarten des olympischen Traums kennenlernen. Und obwohl ich ihn mir schon mehrfach erfüllt habe, ist er immer noch sehr präsent. Olympische Spiele sind einfach das Nonplusultra im Sport. Zum einen ist gerade im Hockey die Leistungsdichte durch die begrenzte Anzahl der Teams unglaublich hoch und schon die Teilnahme an den Olympischen Spielen stellt einen großen Erfolg dar. Zum anderen aber lebt für mich das Flair Olympischer Spiele von der Begegnung mit anderen: Die Zeit im Olympischen Dorf, wo wir unter Gleichgesinnten leben, habe ich immer sehr genossen. Die große Offenheit, die ich dort zum einen sportartübergreifend im deutschen Team, zum anderen aber auch im internationalen Kontakt mit anderen Nationen erlebt habe, ist für mich einzigartig. Die Sportler*innen im Olympischen Dorf haben alle einen langen Weg mit vielen Höhen und Tiefen hinter sich. Verzicht und Disziplin, aber auch eine große Leidenschaft für ihre jeweiligen Sportarten sind Aspekte, die alle miteinander teilen. Der Respekt für die Leistung der anderen ist allgegenwärtig. Egal, aus welcher Kultur man kommt, welchen 
Sport man betreibt oder wie bekannt man ist - alle können sich auf einen gemeinsamen Nenner, das Athlet-Sein, verständigen. Das verbindet.

Natürlich gibt es am Ende auch unglaublich viele, die diesen Weg nicht zu Ende gehen konnten und vorher aufhören. Dem olympischen Sport wird daher ja auch bisweilen vorgeworfen, sich von der Gesellschaft und seinen Wurzeln entfremdet zu haben. Gerade die Athlet"innen aber, die es dann am Ende nach ganz oben geschafft haben, können mit ihren Hero-Geschichten in meinen Augen ein sehr wertvolles Vorbild sein für die ganze Gesellschaft. Das sind junge Menschen, die an ihren Traum glauben und bereit sind, dafür alles in die Waagschale zu werfen, obwohl damit gerade auch finanziell große Einschränkungen verbunden sind. Das finde ich nach wie vor sehr inspirierend.

Diesen positiven Erfahrungen steht im Alltag oft eine ablehnende bzw. geringschätzige Haltung gegenüber, wenn es um Olympische Spiele im Speziellen oder auch um Spitzensport im Allgemeinen geht. Gerade Aspekte wie fehlende Transparenz oder Dopingskandale werfen immer wieder ein schlechtes Licht auf die Olympische Bewegung. In großen deutschen Tageszeitungen entsteht manchmal der Eindruck, Olympische Spiele seien komplett korrupt und überflüssig. Selbstverständlich sind das unglaublich wichtige Aspekte, über die auch berichtet werden muss. Aber Olympische Spiele sind eben auch Schauplatz von Völkerverständigung und Austausch, von sauberen sportlichen Spitzenleistungen und beeindruckenden Fair Play Gesten. Viele Sportler*innen füllen die Erwartungen an ihre Rolle als Vorbilder mit Leben und geben so Kindern und Jugendlichen eine positive Orientierung. Das geht mir zu oft unter, da sollte die Berichterstattung eine Balance finden.

Diese Tendenzen in der öffentlichen Diskussion schlagen sich auch im verhältnismäßig niedrigen Standing nieder, das man als Topathlet*in in Deutschland im Vergleich zu anderen Nationen genießt. Während in den USA Topathlet*innen Stipendien für die besten Unis bekommen, fällt man hier mitunter durch, wenn man wettkampfbedingt eine Klausur nicht mitschreiben kann. Natürlich sollen wir alle vier Jahre unseren Anteil zum Medaillenspiegel beitragen und genießen es dann auch, wenn das ganze Land mit uns mitfiebert. Aber im Anschluss verpufft dieser Effekt oft schnell wieder und die Anerkennung für die Herausforderungen, die man als Leistungssportler"in besonders im Alltag und abseits der öffentlichen Aufmerksamkeit zu bewältigen hat, bleibt in Teilen der Gesellschaft überschaubar.

Als wir in Rio die Bronzemedaille geholt und das im Anschluss in Hamburg gefeiert haben, kam tatsächlich jemand vorbei und meinte: „Ich will mitfeiern, die Chance teilzunehmen habe ja auch ich mit meinen 
Steuergeldern bezahlt.“ Solche Äußerungen, oder wenn dir dann als Olympiateilnehmerin eine Unterschriftenliste gegen Olympia in Hamburg vorgelegt wird, machen uns Athlet*innen auch durchaus zu schaffen. Dabei haben wir so tolle Spitzensportler*innen in Deutschland. Wir müssten das auch als Gesellschaft viel stärker herausstellen und das Potenzial dieser positiven Geschichten besser nutzen, um die olympischen Werte in die Gesellschaft zu tragen.

Bei allen Unsicherheiten, die der Job als Leistungssportlerin und die Jagd nach dem olympischen Traum mit sich bringt: Ich würde mich jederzeit wieder dafür entscheiden. Wenn ich reflektiere, was mich als Mensch geformt hat und wo ich für mein Leben auch nach der Karriere am meisten mitgenommen habe, dann sind das nicht die Seminare in der Uni, Bachelor oder MBA. Da stehen ganz klar die Erfahrungen aus dem Sport, der Umgang mit Siegen und Niederlagen oder Kritik, verinnerlichte Werte wie Fair Play und Respekt oder Begegnungen in internationalen Kontexten wie dem Olympischen Dorf an erster Stelle.

Das letzte Jahr im Lockdown hat noch einmal einen ziemlichen Einschnitt bedeutet. In dieser Unterbrechung des Alltags habe ich wieder deutlich gespürt, wie sehr ich das liebe, was ich mache. Bei aller Unsicherheit, die die Vorbereitung auf Tokio begleitete: Es gab so viel Positives auf dem Weg dorthin, dass es sich hundertprozentig gelohnt hat, noch einmal vollen Einsatz zu bringen, auf Dinge zu verzichten und sich durchzubeißen. Schon auf dem Weg zu den Spielen konnte ich meinen Traum leben! 
